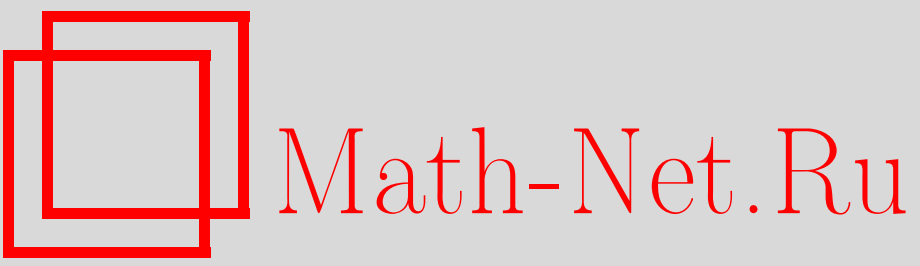

А. О. Смирнов, Эллиптические по $t$ решения нелинейного уравнения Шредингера, ТМФ, 1996, том 107, номер 2, 188-200

DOI: https://doi.org/10.4213/tmf1148

Использование Общероссийского математического портала Math-Net.Ru подразумевает, что вы прочитали и согласны с пользовательским соглашением

http://www . mathnet.ru/rus/agreement

Параметры загрузки:

IP: 3.93 .64 .190

26 апреля 2023 г., $17: 22: 33$ 
ТЕОРЕТИЧЕСКАЯ

И МАТЕМАТИЧЕСКАЯ

ФИЗИКА

Том 107, № 2

май, 1996

А.О. Смирнов

\section{ЭЛЛИПТИЧЕСКИЕ ПО $t$ РЕШЕНИЯ НЕЛИНЕЙНОГО УРАВНЕНИЯ ШРЕДИНГЕРА}

Рассмотрено четыре различных анзаца кривых Кричевера, ассоциированных с эллиптическими по $t$ конечнозонными решениями нелинейного уравнения Шредингера. Приведен пример.

\section{ВВЕДЕНИЕ}

Нелинейное уравнение Шредингера (НШ) наряду с уравнениями Кортевега-де Фриза $($ Кд $\Phi)$, цепочки Тоды и уравнением sine-Гордон играет большую роль при описании многочисленных нелинейных интегрируемых процессов и явлений в физике и математике [1-6]. Значительную часть из них занимают периодические процессы. Поэтому вполне очевидно, насколько важной является задача отыскания периодических решений интегрируемых нелинейных уравнений. И если для уравнения $\mathrm{K}$ д $\Phi[1,7-22]$, цепочки Тоды $[1,23-26]$ и уравнения sine-Гордон $[1,10-12,18,27-29]$ уже найдены периодические по $x$ (по $n$ ) и/или по $t$ конечнозонные решения, то в случае НШ - только по $x[11,12,26]$.

Как обычно, мы будем рассматривать систему нелинейных дифференциальных уравнений

$$
\left\{\begin{aligned}
i p_{t}+p_{x x}-8 p^{2} q & =0 \\
i q_{t}-q_{x x}+8 p q^{2} & =0
\end{aligned}\right.
$$

частным случаем которой (при $q= \pm p^{*}$ ) является НШ

$$
i p_{t}+p_{x x} \mp 8|p|^{2} p=0
$$

В данной работе описываются четыре новых класса двоякопериодических по $t$ решений уравнения НШ (0.1).

Автор благодарит В.Б.Матвеева, А.Р.Итса и Л.А.Бордаг за внимание к работе и полезные обсуждения.

Автор также благодарен Академии естественных наук, Международному научному фонду и Фонду Сороса за поддержку проводимых им исследований. 


\section{1. КОНЕЧНОЗОННЫЕ РЕШЕНИЯ НЕЛИНЕЙНОГО УРАВНЕНИЯ ШРЕДИНГЕРА}

Обшие конечнозонные решения уравнения НШ давно и хорошо известны $[30-34,26]$. Они строятся по гиперэллиптической поверхности (ГП) рода $g$ :

$$
\Gamma: \quad w^{2}=\prod_{j=1}^{2 g+2}\left(E-E_{j}\right),
$$

точки ветвления которой $\left(E=E_{j}, j=1, \ldots, 2 g+2\right)$ являются краями зон спектра оператора Дирака

$$
\left(\begin{array}{cc}
-i & 0 \\
0 & i
\end{array}\right) \boldsymbol{\Psi}_{x}+\left(\begin{array}{cc}
0 & 2 p \\
2 q & 0
\end{array}\right) \boldsymbol{\Psi}=E \boldsymbol{\Psi} .
$$

Бесконечно удаленной точке спектра соответствуют две различные точки данной ГП.

Выберем на Г базис циклов $\gamma^{t}=\left(a_{1}, \ldots, a_{g}, b_{1}, \ldots, b_{g}\right)$ с матрицей пересечения $C_{0}=$ $\left(\begin{array}{rr}0 & \mathrm{I} \\ -\mathrm{I} & 0\end{array}\right)$. Ему соответствует нормированный базис голоморфных дифференциалов $d \mathcal{U}_{j}$,

$$
\oint_{a_{k}} d \mathcal{U}_{j}=\delta_{k j}, \quad k, j=1, \ldots, g
$$

Хорошо известно, что матрица периодов

$$
B_{j k}=\oint_{b_{j}} d \mathcal{U}_{k}, \quad j, k=1, \ldots, g,
$$

есть симметрическая матрица с положительно-определенной мнимой частью.

По матрице периодов $B$ построим тэта-функцию с характеристиками $\boldsymbol{\alpha}, \boldsymbol{\beta} \in \mathbb{R}^{g}$ :

$$
\begin{aligned}
& \Theta[\boldsymbol{\alpha} ; \boldsymbol{\beta}](\mathbf{z} \mid B) \equiv \Theta[\boldsymbol{\alpha} ; \boldsymbol{\beta}](\mathbf{z})= \\
& \quad=\sum_{\mathbf{m} \in \mathbb{Z} g} \exp \{\pi i\langle B(\mathbf{m}+\boldsymbol{\alpha}), \mathbf{m}+\boldsymbol{\alpha}\rangle+2 \pi i\langle\mathbf{m}+\boldsymbol{\alpha}, \mathbf{z}+\boldsymbol{\beta}\rangle\} \\
& \Theta[\mathbf{0} ; \mathbf{0}](\mathbf{z} \mid B) \equiv \Theta(\mathbf{z} \mid B) \equiv \Theta(\mathbf{z})
\end{aligned}
$$

где $\mathbf{z} \in \mathbb{C}^{g}$, суммирование ведется по целочисленной $g$-мерной решетке, а угловые скобки $\langle$,$\rangle обозначают евклидово скалярное произведение.$

Введем на $\Gamma$ нормированные абелевы интегралы второго $-\Omega_{1}(\mathcal{P}), \Omega_{2}(\mathcal{P})$, и третьего $-\omega(\mathcal{P})$ рода, $\mathcal{P} \in \Gamma, \mathcal{P}=(w, E)$, с асимптотикой в бесконечно удаленных точках $\mathcal{P}_{\infty}^{ \pm}$:

$$
\begin{array}{ll}
\oint_{a_{k}} d \Omega_{1}=\oint_{a_{k}} d \Omega_{2}=\oint_{a_{k}} d \omega=0, & k=1, \ldots, g, \\
\Omega_{1}(\mathcal{P})= \pm i\left(E+K_{1}+R_{1} E^{-1}+O\left(E^{-2}\right)\right), & \mathcal{P} \longrightarrow \mathcal{P}_{\infty}^{ \pm}, \\
\Omega_{2}(\mathcal{P})=\mp i\left(2 E^{2}-K_{2}+O\left(E^{-1}\right)\right), & \mathcal{P} \longrightarrow \mathcal{P}_{\infty}^{ \pm}, \\
\omega(\mathcal{P})=\mp\left(\ln E-\ln K_{0}+O\left(E^{-1}\right)\right), & \mathcal{P} \longrightarrow \mathcal{P}_{\infty}^{ \pm}, \\
w= \pm\left(E^{g+1}+O\left(E^{g}\right)\right), & \mathcal{P} \longrightarrow \mathcal{P}_{\infty}^{ \pm} .
\end{array}
$$


Если мы теперь обозначим через $2 \pi i \mathbf{U}, 2 \pi i \mathbf{V}$ векторы $b$-периодов абелевых интегралов второго рода $\Omega_{1}(\mathcal{P})$ и $\Omega_{2}(\mathcal{P})$, соответственно, то функции

$$
\begin{gathered}
p(x, t)=A K_{0} \frac{\Theta(\mathbf{U} x+\mathbf{V} t-\mathbf{Z}-\mathbf{D})}{\Theta(\mathbf{U} x+\mathbf{V} t-\mathbf{Z})} \exp \{i \Phi(x, t)\} \\
q(x, t)=\frac{K_{0}}{A} \frac{\Theta(\mathbf{U} x+\mathbf{V} t-\mathbf{Z}+\mathbf{D})}{\Theta(\mathbf{U} x+\mathbf{V} t-\mathbf{Z})} \exp \{-i \Phi(x, t)\} \\
\Phi(x, t)=2 K_{1} x+2 K_{2} t, \quad \mathbf{D}=\mathcal{U}\left(\mathcal{P}_{\infty}^{+}\right)-\mathcal{U}\left(\mathcal{P}_{\infty}^{-}\right), \\
\mathbf{Z}=\mathcal{K}+\sum_{j=1}^{g} \mathcal{U}\left(\mathcal{P}^{j}\right)
\end{gathered}
$$

для любого $A \neq 0$ будут являться решением системы (0.1). Здесь $\mathcal{K}$ - вектор римановых констант [33]; $\mathcal{P}^{j}, j=1, \ldots, g,-$ неспециальньй дивизор. При этом будет справедливым соотношение [30-33]

$$
4 p q=-\partial_{x}^{2} \ln \Theta(\mathbf{U} x+\mathbf{V} t-\mathbf{Z})-2 R_{1}
$$

которое обобшает на случай $g>1$ известную формулу связи между эллиптическими функциями Якоби и Вейерштрасса.

\section{2. БИРАЦИОНАЛЬНЫЕ ПРЕОБРАЗОВАНИЯ РИМАНОВЫХ ПОВЕРХНОСТЕЙ}

Рассмотрим конечнозонное решение уравнения НШ, построенное по поверхности $\widetilde{\Gamma}$, бирационально эквивалентной Г и имеющей те же самые бесконечно удаленные точки:

$$
\begin{gathered}
\widetilde{\Gamma}: \quad \widetilde{w}^{2}=\prod_{j=1}^{2 g+2}\left(\widetilde{E}-\widetilde{E}_{j}\right), \\
\widetilde{w}=\rho^{g+1} w, \quad \widetilde{E}=\rho E+\delta, \quad \widetilde{E}_{j}=\rho E_{j}+\delta .
\end{gathered}
$$

Легко видеть, что векторы $b$-периодов нормированных абелевых дифференциалов второго рода (1.3), определенных на поверхностях $\widetilde{\Gamma}$ и $Г$, связаны соотношениями

$$
\widetilde{\mathbf{U}}=\rho \mathbf{U}, \quad \widetilde{\mathbf{V}}=\rho^{2} \mathbf{V}-4 \delta \rho \mathbf{U},
$$

из которых нетрудно вывести закон преобразования решений уравнения НШ при растяжении и сдвиге спектра

$$
\begin{aligned}
& \widetilde{p}(x, t)=\rho p\left(\rho x-4 \delta \rho t, \rho^{2} t\right) \exp \left(2 i \delta x+4 \delta^{2} t\right) \\
& \widetilde{q}(x, t)=\rho q\left(\rho x-4 \delta \rho t, \rho^{2} t\right) \exp \left(-2 i \delta x-4 \delta^{2} t\right) .
\end{aligned}
$$

Равенства (2.3) с очевидностью указывают на сушественное различие меж ду такими свойствами решений уравнения НШ, как периодичность по $x$ и периодичность по $t$.

В самом деле, если какое-либо произведение решений $p(x, t) q(x, t)$ периодично по $x$, то и все остальные произведения решений $\widetilde{p} \widetilde{q}$, ассоциированные с бирационально эквивалентными ГП $(2.1)$, будут так же периодическими по $x$. Таким образом, свойство 
$x$-периодичности связано с целым классом бирационально эквивалентных гиперэллиптических поверхностей.

Напротив, если есть некоторое периодичное по $t$ конечнозонное решение уравнения НШ, то в обшем случае ни одно из новых решений $\widetilde{p} \widetilde{q}$ при $\delta \neq 0$ уже не будет обладать этим свойством. Исключение составляют лишш периодические и по $x$, и по $t$ решения, для которых сушествует счетное множество так же периодических и по $x$, и по $t$ решений $\tilde{p q}$, т.е. $t$-периодичность конечнозонного решения соответствует не всем бирационально эквивалентным ГП $\widetilde{\Gamma}$, а только одной из них или счетному их множеству.

\section{3. КРИВЫЕ КРИЧЕВЕРА. АНЗАЦ I}

Рассмотрим риманову поверхность $\Gamma=\{(k, \alpha)\}$ рода $g, n$-листно, $n \geqslant 3$, накрывающую тор $\Gamma_{0}$ :

$$
\Gamma_{0}:\left\{\wp^{\prime}(\alpha)\right\}^{2}=4 \wp^{3}(\alpha)-g_{2} \wp(\alpha)-g_{3} \equiv 4 \prod_{j=1}^{3}\left(\wp(\alpha)-e_{j}\right)
$$

и удовлетворяющую следуюшим требованиям: 1)

$$
\Gamma: \mathcal{R}(k, \alpha) \equiv k^{n}+\sum_{j=2}^{n} r_{j}(\alpha) k^{n-j}=0
$$

где $r_{j}(\alpha)$ - эллиптические функции от $\alpha \in \Gamma_{0}$ с полюсами в точках $\alpha= \pm \alpha_{0}$;

$2)$ на $\Gamma$ "над" $\alpha= \pm \alpha_{0}$ лежат две точки ветвления первого порядка $-\mathcal{P} \underset{\infty}{ \pm}$, в окрестности которых функция $k(\alpha)$ имеет асимптотику

$$
k=\mp\left(\frac{n-2}{\xi_{ \pm}^{2}}+\frac{2 n \varkappa}{\xi_{ \pm}}+O(1)\right), \quad \xi_{ \pm}^{2}=\alpha_{0} \mp \alpha,
$$

и других точек ветвления "над" $\alpha= \pm \alpha_{0}$ нет;

3 ) в остальных точках $\Gamma$ "над" $\alpha= \pm \alpha_{0}$ имеем

$$
k=\frac{2}{\alpha_{0} \mp \alpha}+O(1)
$$

4) на Г существует голоморфная инволюция

$$
\tau:(k, \alpha) \longrightarrow(-k,-\alpha), \quad \tau: \xi_{+} \longrightarrow \xi_{-},
$$

имеющая $2 g+2$ неподвижные точки.

ЗАмЕчанИЕ 1. Из п. 4 и 2 следует, что поверхность Г является гиперэллиптической и что пара выделенных точек $\mathcal{P}_{\infty}^{ \pm}$переходят друг в друга при гиперэллиптической инволюции $\tau$.

ЗАмечаниЕ 2. Используя формулу Римана-Гурвица $[35,12]$, нетрудно получить, что род $g$ поверхности Г (3.1)-(3.5) равен $p+2$, где $2 p$ - число нулей функции $\partial_{k} \mathcal{R}(k, \alpha)$, не лежаших "над” $\alpha= \pm \alpha_{0}$ и отличных от нулей функции $\partial_{\alpha} \mathcal{R}(k, \alpha)$ (с учетом кратности). 
Теорема 1. Решения $p(x, t)$ и $q(x, t)$ уравнения НШ $(0.1)$, построенные по ГП $\Gamma_{g}^{n}(3.1)-(3.5)$ и отмеченным точкам $\mathcal{P}_{\infty}^{ \pm}$“над” $\alpha= \pm \alpha_{0}$, могут быть выражсены c точностью до әкспоненциальных множителей через әллиптические б-функции Вейеритрасса (с периодами накрываемой поверхности (3.1)) от $\frac{i n}{8} t$. При этом произведение функиий $p(x, t)$ u $q(x, t)$ будет әллиптической функичей по $\frac{i n}{8} t$.

ДокАЗАТЕЛьство. Выберем в качестве спектральной переменной функцию $E(\mathcal{P})$, $\mathcal{P} \in \Gamma$, инвариантную относительно инволюции $\tau(3.5)$ и имеющую пару простых полюсов в точках $\mathcal{P}_{\infty}^{ \pm}$вида

$$
E=\frac{1}{\xi_{ \pm}}+\varkappa+O\left(\xi_{ \pm}\right), \quad \mathcal{P} \longrightarrow \mathcal{P}_{\infty}^{ \pm}
$$

Из (3.6) вытекает, что нормированный абелев дифференциал второго рода $d \Omega_{2}$ будет иметь следуюшую асимптотику в окрестности точек $\mathcal{P}_{\infty}^{ \pm}$

$$
d \Omega_{2}=\mp i\left(4 E+O\left(E^{-2}\right)\right) d E= \pm i\left(\frac{4}{\xi_{ \pm}^{3}}+\frac{4 \varkappa}{\xi_{ \pm}^{2}}+O(1)\right) d \xi_{ \pm}
$$

Применяя теорему Лиувилля, нетрудно показать, что нормированные абелевы дифференциалы второго рода $d \Omega_{2}$ и

$$
d \Omega^{0}=-\frac{1}{2}\left(\wp\left(\alpha-\alpha_{0}\right)+\wp\left(\alpha+\alpha_{0}\right)+2 \eta / \omega\right) d \alpha
$$

определенные на $Г$ и $\Gamma_{0}$, соответственно, связаны соотношением

$$
\sigma^{*} d \Omega^{0} \equiv-\frac{i n}{8} d \Omega_{2}-\frac{1}{4} d k
$$

с точностью до голоморфных дифференциалов. Здесь $\sigma$ есть накрываюшее отображение, $\sigma: \Gamma \rightarrow \Gamma_{0}, \sigma(k, \alpha)=\alpha ; 2 \omega, 2 \omega^{\prime}$ - периоды тора $(3.1) ; \eta=\zeta(\omega), \zeta(\alpha)$ - эллиптическая функция Вейерштрасса.

Теперь легко видеть, что утверждение теоремы 1 является следствием теоремы о редукции многомерной тэта-функции, накрывающей эллиптическую $[18,21,22,26,28]$, равенства (3.9) и формул связи эллиптических функций Вейерштрасса с одномерными тэта-функциями [36].

ЗАмечаниЕ 3. Решение уравнения НШ, построенное по гиперэллиптической поверхности, удовлетворяющей условию теоремы 1 (или условию одной из теорем, доказанных ниже), мы будем называть эллиптическим по $t$ решением уравнения НШ.

СЛЕДСТВИЕ 1. Сумма краев зон ГП, ассочиированной $с$ әллиптическим по $t$ решением уравнения НШІ (анзач I), равна

$$
c_{0}=\sum_{j=1}^{6} E_{j}=6 \varkappa .
$$


ДокАЗАТЕЛЬСТво. Из (3.6), (1.1) и (1.3) вытекает, что функция $w(\mathcal{P})$ в окрестности точек $\mathcal{P}_{\infty}^{ \pm}$имеет асимптотику

$$
w= \pm\left\{\frac{1}{\xi_{ \pm}^{g+1}}+\frac{2(g+1) \varkappa-c_{0}}{2 \xi_{ \pm}^{g}}+O\left(\frac{1}{\xi_{ \pm}^{g-1}}\right)\right\}
$$

следовательно,

$$
\sigma^{*} d \alpha=\left\{2 E^{g-2}+\left(6 \varkappa-c_{0}\right) E^{g-3}+\sum_{k=4}^{g} d_{k} E^{g-k}\right\} \frac{d E}{w} .
$$

Полагая $g=2$, получаем равенство

$$
\sigma^{*} d \alpha=2 \frac{d E}{w}
$$

и соотношение (3.10).

ЗАмечАниЕ 4. Можно доказать, что кривые данного типа (для рода $g=2$ ) ассоциированы с двухзонными эллиптическими и по $x$ [26], и по $t$ решениями уравнения НШ. Иначе говоря, анзац I кривых Кричевера, ассоциированных с эллиптическими по $t$ решениями уравнения НШ, является аналогом анзаца I кривых Кричевера, ассоциированных с эллиптическими по $t$ решениями уравнения $\mathrm{K} д \Phi[21,22]$.

\section{4. АНЗАЦ II. ОДНОПАРАМЕТРИЧЕСКОЕ СЕМЕЙСТВО РЕШЕНИЙ}

Рассмотрим риманову поверхность $\Gamma$ рода $g, n$-листно накрываюшую эллиптическую поверхность $\Gamma_{0}$ (3.1) и удовлетворяюшую следуюшим требованиям: 1)

$$
\Gamma: \mathcal{R}(k, \alpha) \equiv k^{n}+A\left\{\wp\left(\alpha+\alpha_{0}\right)-\wp\left(\alpha-\alpha_{0}\right)\right\} k^{n-1}+\sum_{i=2}^{n} r_{i}(\alpha) k^{n-i}=0,
$$

где $A \neq 0$-параметр, $r_{i}(\alpha)$ - эллиптические функции от $\alpha \in \Gamma_{0}$ с полюсами в точках $\alpha= \pm \alpha_{0}$

2 ) в окрестности $\alpha= \pm \alpha_{0}$ функция $\mathcal{R}(k, \alpha)$ представима в виде

$$
\mathcal{R}(k, \alpha)=\left(k \mp \frac{A}{\left(\alpha \mp \alpha_{0}\right)^{2}}+\frac{n-1}{\alpha \mp \alpha_{0}}+b_{n}^{ \pm}\left(\alpha \mp \alpha_{0}\right)\right) \prod_{j=1}^{n-1}\left(k-\frac{1}{\alpha \mp \alpha_{0}}+b_{j}^{ \pm}\left(\alpha \mp \alpha_{0}\right)\right),
$$

где $b_{j}^{ \pm}\left(\alpha \mp \alpha_{0}\right)$ - регулярные функции $\alpha \mp \alpha_{0}$;

3) на Г существует инволюция

$$
\tau:(k, \alpha) \longrightarrow(-k,-\alpha),
$$

имеюшая $2 g+2$ неподвижные точки.

ЗАмЕчАниЕ 5. Род $g$ поверхности Г (3.1), (4.1)-(4.3) равен $p+1$, где $2 p$ - число нулей функции $\partial_{k} \mathcal{R}(k, \alpha)$, не лежаших “над" $\alpha= \pm \alpha_{0}$ и отличных от нулей функции $\partial_{\alpha} \mathcal{R}(k, \alpha)$ (с учетом кратности). 
Теорема 2. Решения $p(x, t)$ и $q(x, t)$ уравнения НШ $(0.1)$, построенные по ГП $\Gamma_{g}^{n}(3.1),(4.1)-(4.3)$ и отмеченным точкам $\mathcal{P}_{\infty}^{ \pm}$“над” $\alpha= \pm \alpha_{0}$, могут бить виражены с точностью до әкспоненииальных множителей через эллиптические $\sigma$-функиии Вейеритрасса ( с периодами накрываемой поверхности (3.1)) от $\frac{i A}{4} t$. При этом произведение функций $p(x, t)$ и $q(x, t)$ будет әллиптической функиией no $\frac{i A}{4} t$.

ДокАЗАТЕЛЬство. Как и ранее, выберем в качестве спектральной переменной функцию $E(\mathcal{P}), \mathcal{P} \in \Gamma$, инвариантную относительно инволюции $\tau(4.3)$ и имеющую пару простых полюсов в точках $\mathcal{P}_{\infty}^{ \pm}$:

$$
E=\frac{1}{\alpha_{0} \mp \alpha}+\frac{n}{2 A}+O\left(\alpha_{0} \mp \alpha\right), \quad \mathcal{P} \longrightarrow \mathcal{P}_{\infty}^{ \pm}
$$

Такой выбор функции $E(\mathcal{P})$ приводит к тому, что нормированный абелев дифференциал второго рода $d \Omega_{2}$ в окрестности точек $\mathcal{P} \underset{\infty}{ \pm}$ будет иметь следуюшую асимптотику:

$$
\begin{aligned}
d \Omega_{2} & =\mp i\left(4 E+O\left(E^{-2}\right)\right) d E= \\
& =-i\left(\frac{4}{\left(\alpha_{0} \mp \alpha\right)^{3}}+\frac{2 n}{A\left(\alpha_{0} \mp \alpha\right)^{2}}+O(1)\right) d \alpha .
\end{aligned}
$$

Легко видеть, что нормированные абелевы дифференциалы второго рода $d \Omega_{2}$ и $d \Omega^{0}$ $(3.8)$, определенные на $Г$ и $\Gamma_{0}$, соответственно, связаны с точностью до голоморфных дифференциалов соотношением

$$
\sigma^{*} d \Omega^{0} \equiv-\frac{i A}{4} d \Omega_{2}+\frac{1}{2} d k
$$

которое и доказывает утверждение теоремы 2.

ЗАмЕЧАнИЕ 6 . При указанном выше выборе мероморфной функции $E(\mathcal{P})$ функция $w(\mathcal{P})$ в окрестности точек $\mathcal{P}_{\infty}^{ \pm}$имеет асимптотику

$$
w= \pm\left\{\frac{1}{\left(\alpha_{0} \mp \alpha\right)^{g+1}}+\frac{n(g+1)-A c_{0}}{2 A\left(\alpha_{0} \mp \alpha\right)^{g}}+O\left(\frac{1}{\left(\alpha_{0} \mp \alpha\right)^{g-1}}\right)\right\}
$$

Следовательно, накрытия, удовлетворяющие теореме 2, соответствуют редукции голоморфного гиперэллиптического интеграла вида

$$
\sigma^{*} d \alpha=\left\{E^{g-1}+\frac{2 n-A c_{0}}{2 A} E^{g-2}+\sum_{k=3}^{g} f_{k} E^{g-k}\right\} \frac{d E}{w}
$$

Сравнивая (3.12) и (4.8), мы видим, что анзацы I и II отличаются друг от друга редуцируемыми интегралами. 


\section{5. ОСОБЫЙ СЛУЧАЙ. АНЗАЦ III}

Легко видеть, что при определении анзаца I значение параметра $\alpha_{0} \notin\left\{0, \omega_{1}, \omega_{2}, \omega_{3}\right\}$. Поэтому случай $\alpha_{0}=0$ надо рассматривать отдельно.

Пусть риманова поверхность Г рода $g \quad n$-листно $(n \geqslant 5)$ накрывает эллиптическую $\Gamma_{0}$ (3.1) и удовлетворяет следуюшим требованиям: 1$)$

$$
\Gamma: \mathcal{R}(k, \alpha) \equiv k^{n}+\sum_{j=2}^{n} r_{j}(\alpha) k^{n-j}=0
$$

где $r_{j}(\alpha)$ - эллиптические функции от $\alpha \in \Gamma_{0}$ с полюсами в точке $\alpha=0$;

$2)$ на $\Gamma$ "над" $\alpha=0$ лежат две точки ветвления первого порядка $-\mathcal{P}_{\infty}^{ \pm}$, в окрестности которых функция $k(\alpha)$ имеет асимптотику

$$
k=\mp\left(\frac{n-4}{\xi_{ \pm}^{2}}+\frac{2 n \varkappa}{\xi_{ \pm}}+O(1)\right), \quad \xi_{ \pm}^{2}=\mp \alpha,
$$

и других точек ветвления "над" $\alpha=0$ нет;

3 в в остальных точках $\Gamma$ "над" $\alpha=0$

$$
k=-\frac{4}{\alpha}+O(1)
$$

4) на Г сушествует голоморфная инволюция

$$
\tau:(k, \alpha) \longrightarrow(-k,-\alpha), \quad \tau: \xi_{+} \longrightarrow \xi_{-},
$$

имеюшая $2 g+2$ неподвижные точки.

ЗАмечаниЕ 7. Род $g$ поверхности Г (3.1), (5.1)-(5.4) равен $p+2$, где $2 p$-число нулей функции $\partial_{k} \mathcal{R}(k, \alpha)$, не лежаших "над" $\alpha=0$ и отличных от нулей функции $\partial_{\alpha} \mathcal{R}(k, \alpha)(c$ учетом кратности).

Tеорема 3. Решения $p(x, t)$ и $q(x, t)$ уравнения НШІ (0.1), построенные по ГП $\Gamma_{g}^{n}$ (3.1), (5.1)-(5.4) и отмеченным точкам $\mathcal{P}_{\infty}^{ \pm}$“над” $\alpha=0$, могут быть выражены с точностью до әкспоненциальных множителей через әллиптические $\sigma$-функиии Вейеритрасса (с периодами накрываемой поверхности (3.1)) от $\frac{i n}{8} t$. При этом произведение функиий $p(x, t)$ u $q(x, t)$ будет әллиптической функцией no $\frac{i n}{8} t$.

ДокАЗАТЕЛЬСтво. Выберем в качестве спектральной переменной функцию $E(\mathcal{P})$, $\mathcal{P} \in \Gamma$, инвариантную относительно инволюции $\tau$ (5.4) и имеющую пару простых полюсов в точках $\mathcal{P}_{\infty}^{ \pm}$вида

$$
E=\frac{1}{\xi_{ \pm}}+\varkappa+O\left(\xi_{ \pm}\right), \quad \mathcal{P} \longrightarrow \mathcal{P}_{\infty}^{ \pm}
$$


Из (5.5) вытекает, что нормированный абелев дифференциал второго рода $d \Omega_{2}$ будет иметь следуюшую асимптотику в окрестности точек $\mathcal{P}_{\infty}^{ \pm}$:

$$
d \Omega_{2}=\mp i\left(4 E+O\left(E^{-2}\right)\right) d E= \pm i\left(\frac{4}{\xi_{ \pm}^{3}}+\frac{4 \varkappa}{\xi_{ \pm}^{2}}+O(1)\right) d \xi_{ \pm} .
$$

Нормированные абелевы дифференциалы второго рода $d \Omega_{2}$ и

$$
d \Omega^{0}=-(\wp(\alpha)+\eta / \omega) d \alpha
$$

определенные на $Г$ и $\Gamma_{0}$, соответственно, связаны соотношением

$$
\sigma^{*} d \Omega^{0} \equiv-\frac{i n}{8} d \Omega_{2}-\frac{1}{4} d k
$$

с точностью до голоморфных дифференциалов, и, таким образом, утверждение теоремы 3 также является доказанным.

СЛЕДСТВИЕ 2. Сумма краев зон ГП, ассоциированной с әллиптическим по $t$ решением уравнения НШІ (анзаи III), равна

$$
c_{0}=\sum_{j=1}^{6} E_{j}=6 \varkappa
$$

ДокАЗАТЕЛЬСтво. Из (5.5), (1.1) и (1.3) вытекает, что функция $w(\mathcal{P})$ в окрестности точек $\mathcal{P}_{\infty}^{ \pm}$имеет асимптотику

$$
w= \pm\left\{\frac{1}{\xi_{ \pm}^{g+1}}+\frac{2(g+1) \varkappa-c_{0}}{2 \xi_{ \pm}^{g}}+O\left(\frac{1}{\xi_{ \pm}^{g-1}}\right)\right\}
$$

следовательно,

$$
\sigma^{*} d \alpha=\left\{2 E^{g-2}+\left(6 \varkappa-c_{0}\right) E^{g-3}+\sum_{k=4}^{g} d_{k} E^{g-k}\right\} \frac{d E}{w} .
$$

Полагая $g=2$, получаем равенство

$$
\sigma^{*} d \alpha=2 \frac{d E}{w}
$$

и соотношение $(5.9)$.

ЗАМЕчАнИЕ 8 . Нетрудно доказать, что кривые рода $g=2$ ассоциированы с двухзонными эллиптическими и по $x$ ([26], анзац I), и по $t$ решениями уравнения НШ. Поскольку оба накрытия для кривой рода 2 имеют одинаковое число листов [35], минимальное число листов для кривой Кричевера, по которой может быть построено двухзонное эллиптическое по $x$ решение уравнения НШ (анзац I), так же должно быть равно $n=5$, что объясняет соответствуюшие результаты работы [26]. 


\section{6. АНЗАЦ IV. ОДНОПАРАМЕТРИЧЕСКОЕ СЕМЕЙСТВО РЕШЕНИЙ. ОСОБЫЙ СЛУЧАЙ}

Рассмотрим риманову поверхность $\Gamma$ рода $g, n$-листно накрываюшую $(n \geqslant 3)$ эллиптическую поверхность $\Gamma_{0}$ (3.1) и удовлетворяюшую следуюшим требованиям: 1$)$

$$
\Gamma: \mathcal{R}(k, \alpha) \equiv k^{n}+\sum_{i=2}^{n} r_{i}(\alpha) k^{n-i}=0,
$$

где $r_{i}(\alpha)$ - эллиптические функции от $\alpha \in \Gamma_{0}$ с полюсами в точке $\alpha=0$;

2 ) в окрестности $\alpha=0$ функция $\mathcal{R}(k, \alpha)$ представима в виде

$$
\begin{aligned}
\mathcal{R}(k, \alpha)= & \left(k-\frac{A}{\alpha^{2}}+\frac{n-2}{\alpha}+b_{n}(\alpha)\right) \times \\
& \times\left(k+\frac{A}{\alpha^{2}}+\frac{n-2}{\alpha}-b_{n}(-\alpha)\right) \prod_{j=1}^{n-2}\left(k-\frac{2}{\alpha}+b_{j}(\alpha)\right),
\end{aligned}
$$

где $b_{j}(\alpha)$ - регулярные функции $\alpha$;

3) на Г сушествует инволюция

$$
\tau:(k, \alpha) \longrightarrow(-k,-\alpha),
$$

имеюшая $2 g+2$ неподвижные точки.

ЗАмЕчАнИЕ 9. Род $g$ поверхности Г (3.1), (6.1)-(6.3) равен $p+1$, где $2 p$-число нулей функции $\partial_{k} \mathcal{R}(k, \alpha)$, не лежащих "над" $\alpha=0$ и отличных от нулей функции $\partial_{\alpha} \mathcal{R}(k, \alpha)$ (с учетом кратности).

Tеорема 4. Решения $p(x, t)$ и $q(x, t)$ уравнения НШШ (0.1), построенные по ГП $\Gamma_{g}^{n}$ (3.1), (6.1)-(6.3) и отмеченнымм точкам $\mathcal{P}_{\infty}^{ \pm}$“над” $\alpha=0$, могут быть выражены с точностью до әкспоненциальных множителей через әллиптические б-функци Вейеритрасса ( с периодами накрываемой поверхности (3.1)) от $\frac{i A}{4} t$. При этом произведение функций $p(x, t)$ и $q(x, t)$ будет әллиптической функиией no $\frac{i A}{4} t$.

ДокАЗАТЕЛЬСтво. Как и ранее, выберем в качестве спектральной переменной функцию $E(\mathcal{P}), \mathcal{P} \in \Gamma$, инвариантную относительно инволюции $\tau$ (6.3) и имеюшую пару простых полюсов в точках $\mathcal{P}_{\infty}^{ \pm}$,

$$
E=\mp \frac{1}{\alpha}+\frac{n}{2 A}+O(\alpha), \quad \mathcal{P} \longrightarrow \mathcal{P}_{\infty}^{ \pm} .
$$

Такой выбор функции $E(\mathcal{P})$ приводит к тому, что нормированный абелев дифференциал второго рода $d \Omega_{2}$ в окрестности точек $\mathcal{P} \underset{\infty}{ \pm}$ будет иметь следуюшую асимптотику:

$$
d \Omega_{2}=\mp i\left(4 E+O\left(E^{-2}\right)\right) d E=i\left( \pm \frac{4}{\alpha^{3}}-\frac{2 n}{A \alpha^{2}}+O(1)\right) d \alpha .
$$

Легко видеть, что нормированные абелевы дифференциалы второго рода $d \Omega_{2}$ и $d \Omega^{0}$ (5.7), определенные на $\Gamma$ и $\Gamma_{0}$, соответственно, связаны с точностью до голоморфных дифференшиалов соотношением

$$
\sigma^{*} d \Omega^{0} \equiv-\frac{i A}{4} d \Omega_{2}+\frac{1}{2} d k
$$

которое и доказывает утверждение теоремы 4. 
ЗАМЕЧАНИЕ 10. При указанном выше выборе мероморфной функции $E(\mathcal{P})$ функция $w(\mathcal{P})$ в окрестности точек $\mathcal{P}_{\infty}^{ \pm}$имеет асимптотику

$$
w=(\mp 1)^{g}\left\{-\frac{1}{\alpha^{g+1}} \pm \frac{n(g+1)-A c_{0}}{2 A \alpha^{g}}+O\left(\frac{1}{\alpha^{g-1}}\right)\right\} .
$$

Таким образом, накрытия, удовлетворяющие теореме 4, соответствуют редукции гиперэллиптического интеграла

$$
\sigma^{*} d \alpha=\left\{E^{g-1}+\frac{2 n-A c_{0}}{2 A} E^{g-2}+\sum_{k=3}^{g} f_{k} E^{g-k}\right\} \frac{d E}{w}
$$

\section{7. ПРИМЕР}

Кривая $\Gamma_{2}^{3}=\{(k, \alpha)\}$ (анзац IV) определяется уравнением

$$
k^{3}-A^{2}\left(\wp(\alpha)-e_{j}\right)(\wp(\alpha)+B) k-A^{2}\left(\wp(\alpha)-e_{j}\right) \wp^{\prime}(\alpha)=0,
$$

где $e_{j}=4 r^{2} t^{2}$

$$
A^{-1}=2 t s(1-s), \quad B=2 r^{2} t^{2}+2 t^{2}(2 s-1)^{2}\left(2 s^{2}-2 s-1\right)
$$

$r, s, t$ - вспомогательные параметры решения,

$$
e_{k}=2 t^{2}(1-2 s)^{3}-2 t^{2} r^{2}, \quad e_{l}=2 t^{2}(2 s-1)^{3}-2 t^{2} r^{2}
$$

Каноническая форма гиперэллиптической кривой Г⿸厂

$$
w^{2}=\left(E-E_{1}\right)\left(E-E_{2}\right)\left(E-E_{3}\right)\left(E^{3}+A_{1} E^{2}+B_{1} E+C_{1}\right),
$$

где

$$
\begin{aligned}
E_{1}= & -t\left(s^{2}+3 s-2\right), \quad E_{2}=-t\left(s^{2}-5 s+2\right), \\
E_{3}= & -t\left(5 s^{2}-5 s+2\right), \quad A_{1}=t\left(11 s^{2}-11 s+2\right), \\
B_{1}= & -6 t^{2} r^{2}+t^{2}\left(35 s^{4}-70 s^{3}+35 s^{2}-2\right), \\
C_{1}= & -6 t^{3} r^{2}\left(5 s^{2}-5 s+2\right)+ \\
& \quad+t^{3}\left(5 s^{3}+s^{2}-6 s+2\right)\left(5 s^{3}-16 s^{2}+11 s-2\right) .
\end{aligned}
$$

Связь между различными представлениями кривой $\Gamma_{2}^{3}$ осушествляется с помощью соотношений

$$
\begin{aligned}
\wp^{\prime}(\alpha) & =\frac{2 w\left(E-H_{1}\right)\left(E-H_{2}\right)}{\left(E-E_{3}\right)^{2}}, \quad k=\frac{A w}{E-E_{3}}, \\
\wp(\alpha) & =\frac{E^{3}+A_{2} E^{2}+B_{2} E+C_{2}}{E-E_{3}},
\end{aligned}
$$


где $A_{2}=A_{1}, B_{2}=B_{1}+e_{j}$,

$$
\begin{aligned}
H_{1}= & -t(s-1)(5 s-2), \quad H_{2}=-t s(5 s-3), \\
C_{2}= & -2 t^{3} r^{2}\left(5 s^{2}-5 s+2\right)+ \\
& +t^{3}\left(5 s^{3}+s^{2}-6 s+2\right)\left(5 s^{3}-16 s^{2}+11 s-2\right) .
\end{aligned}
$$

Накрытие (6.1), (3.1) соответствует редукции ненормированного голоморфного диффференциала

$$
d \alpha=\frac{E-H_{3}}{w} d E
$$

и ненормированного абелева дифференциала второго рода с полюсами второго порядка в точках $\mathcal{P}_{\infty}^{ \pm}$

$$
\begin{aligned}
d \Omega_{2} & =-\frac{2 i}{A}(2 \wp(\alpha) d \alpha+d k)= \\
& =-4 i\left\{E^{4}+A_{3} E^{3}+B_{3} E^{2}+C_{3} E+D_{3}\right\} \frac{d E}{w},
\end{aligned}
$$

где

$$
\begin{aligned}
H_{3} & =-t\left(3 s^{2}-3 s+2\right), \quad A_{3}=t(3 s-1)(3 s-2), \\
B_{3} & =-3 t^{2} r^{2}+3 t^{2}\left(7 s^{4}-14 s^{3}+5 s^{2}+2 s-1\right), \\
C_{3} & =-2 t^{3} r^{2}\left(7 s^{2}-7 s+3\right)+ \\
& +t^{3}\left(3 s^{6}-9 s^{5}-77 s^{4}+169 s^{3}-132 s^{2}+46 s-6\right), \\
D_{3} & =t^{4} r^{2} s(1-s)\left(3 s^{2}-3 s-2\right)+ \\
& +t^{4} s(1-s)\left(10 s^{6}-30 s^{5}+108 s^{4}-166 s^{3}+121 s^{2}-43 s+6\right) .
\end{aligned}
$$

\section{Список литературы}

[1] Захаров В. Е., Манаков С. В., Новиков С. П., Питаевский Л. П. Теория солитонов: метод обратной задачи. М.: Наука, 1980.

[2] Тахтаджян Л. А., Фаддеев Л. Д. Гамильтонов подход в теории солитонов. М.: Наука, 1986.

[3] Калоджеро Ф., Дегасперис А. Спектральные преобразования и солитоны. М.: Мир, 1985.

[4] Абловии, M., Сигур X. Солитоны и метод обратной задачи. М.: Мир, 1987.

[5] Додд P., Эйлбек Дж., Гиббон Дж., Моррис Х. Солитоны и нелинейные волновые уравнения. М.: Мир, 1988.

[6] Нъюәлл A. Солитоны в математике и физике. М.: Мир, 1989.

[7] Дубровин Б. А., Новиков С. П. // ЖЭТФ. 1974. Т. 67. №12. С. 2131-2143.

[8] Airault H., McKean H. P., Moser J. // Commun. Pure Appl. Math. 1977. V. 30. P. 95-148.

[9] Кричевер И. М. // Функц. анализ и его прилож. 1980. Т. 14. № 4. С. 45-54.

[10] Белоколос Е. Д., Энольский В. З. // ТМФ. 1982. Т. 53. № 2. С. 271-282.

[11] Бабич М. В., Бобенко А. И., Матвеев В. Б. // Изв. АН СССР, сер. матем. 1985. Т. 49. №3. C. $511-529$.

[12] Белоколос Е. Д., Бобенко А. И., Матвеев В. Б., Энольский В. З. // УМН. 1986. Т. 41. № 2. C. $3-42$.

[13] Verdier J. -L. // Algebraic Analysis. Vol. II (Micio Sato Sixtieth Birthday Vol.): Academic Press, 1988.. P. 901-910.

[14] Treibich A. // Duke Math. J. 1989. V. 59. № 3. P. 611-627. 
[15] Treibich A., Verdier J. - L. Solitons elliptiques (Volume en l'honneur du $60^{e}$ anniversaire du prof. A. Grothendieck). Boston: Birkhäuser, 1990.

[16] Белоколос Е. Д., Энольский В. З. // УМН. 1989. Т. 44. № 5. С. 155-156.

[17] Белоколос Е. Д., Энольский В. З. // Функц. анализ и его прилож. 1989. Т. 23. № 1. С. 57-58.

[18] Смирнов А. О. // Мат. заметки. 1989. Т. 46. № 5. С. 100-102.

[19] Смирнов А. О. // Мат. заметки. 1989. Т. 45. №6. С. 66-73.

[20] Тайманов И. А. // ТМФ. 1990. Т. 84. №1. С. 38-45.

[21] Смирнов А. О. // ТМФ. 1994. Т. 100. № 2. С. 183-198.

[22] Smirnov A. O. // Acta Appl. Math. 1994. V. 36. P. 125-166.

[23] Кричевер И. М. // УМН. 1978. Т. 33. № 4. С. 215-216.

[24] Кричевер И. М. // УМН. 1981. Т. 36. № 2. С. 79-80.

[25] Смирнов А. О. // ТМФ. 1989. Т. 78. №1. С. 11-21.

[26] Смирнов А. О. // Мат. сборник. 1994. Т. 185. № 8. С. 103-114.

[27] Бобенко А. И. // Функц. анализ и его прилож. 1984. Т. 18. № 3. С. 74-75.

[28] Смирнов А. О. // Мат. сборник. 1990. Т. 181. №6. С. 804-812.

[29] Смирнов A. O. // Вопросы квантовой теории поля и статистической физики (Зап. научн. семин. ПОМИ. Т. 205). С. -Пб.: Наука, 1993.

[30] Итс А. Р. // Вестн. ЛГУ, сер. мат.-мех.-астр. 1976. № 7. Вып. 2. С. 39-46.

[31] Иmс A. P. Точное интегрирование в римановых $\Theta$-функциях нелинейного уравнения Шредингера и модифицированного уравнения Кортевега-де Фриза: Дис. канд. физ. -матем. наук. Л.: ЛГУ, 1977.

[32] Итс А. Р., Котляров В. П. // ДАН УССР, сер. А. 1976. №11. С. 965-968.

[33] Matveev V. B. Abelian functions and solitons: Preprint № 373. Univ. of Wrocław, 1976.

[34] Итс А. Р. // Изв. АН СССР, сер. матем. 1985. Т. 49. № 3. С. 530-565.

[35] Krazer A. Lehrbuch der Thetafunktionen. Leipzig: Teubner, 1903.

[36] Ахиезер Н. И. Элементы теории эллиптических функций. М.: Наука, 1970.

Санкт-Петербургская государственная академия аэрокосмического приборостроения

Поступила в редакцию 27.III.1995 г.

\section{A. O. Smirnov \\ ELLIPTIC IN $t$ SOLUTIONS OF THE NONLINEAR SCHRÖDINGER EQUATION}

Four various ansatz of the Krichever curves for the elliptic in $t$ solutions of the nonlinear Schrödinger equation are considered. An example is given. 